\title{
Contents, Vol. 124, 1952
}

\section{Index}

Althaus, H., vide E. Weigelin.

Appelmans, M., et J. Blocked, Examen du vitré au microscope électronique

Barth, J., Ein Spezialgerät zur Augenphotographie . . . 172

Bischoff, H., Ein Beitrag zur Gewebstherapie nach Filatow. (Beobachtungen an 90 mit subkonjunktivalen Placen-tar-Implantationen behandelten Kranken.) .... 89

Blocked, J., vide M. Appelmans.

Brand, I., A new Instrument to Assist in Intracapsular Cataract Extraction through a Round Pupil .... 108

Cazaban, R., vide C. Gros.

Deweer, J. P., vide J. Francois.

Ercolí, G., and G. Leprí, Dacryosialoadenopathia Atrophi-

cans and Sclerodermia

Fazakas, A., und A. Szauter, Diagnostische Bedeutung derim Augenhintergrund bef indlichen perivaskulären Binde-gewebsstränge, mit besonderer Berücksichtigung derEpi-lepsie 303 Francois, J., et G. Verríest. Rétinopathie pigmentaire unilatérale 65

- $\quad$ et /. P. Deweer, Sclérose tubéreuse cérébrale de Bourne-

ville 321

Gros, C., et R. Cazaban, Papillites et indications neuro-chi-

rurgicales 197

Hager, H., Irgapyrin-Therapie bei Augenerkrankungen . 205Halbertsma, K. T. A., Coloboma Papillae et Chorioideae Associated with Changes at the Macula 293

Koch, C., Über experimentelle Häutchenbildung in Glaskörper und subretinaler Flüssigkeit $\quad 340$

Leprí, G., vide G. Ercoli.

Pau, H., Die akkommodative Linsenverschiebung als Aus-druck antagonist] sch wirkender Kräfte.

I. Mitteilung 239

II. Mitteilung 271

Pavisic, Z., Beitrag zum tarsoplastischen Operationsverfahren gegen Narbenentropium und Trichiasis .... 35

- Untersuchungen über Refraktion und Astigmatismus derHornhaut beim Pterygium 157

Posthumus, R. G., The Use and the Possibilities of Progesterone in the Treatment of Glaucoma 17

Prijot, E., et R. Weekers, Mesure de la resistance à Гécoule-ment de Гhumeur aqueuse au moyen du tonomètre électronique. $4 \mathrm{e}$ partie. Mode d'action de la pilocarpine dans le glaucome chronique non inflammatoire .... 12 
- $\quad$ vide R. Weekers.

Sie-Boen-Lian, Notes on Experimental Transplantation of

Sclera into Cornea. (A preliminary report.) . 287

Símková, M., and K. Zahn, The Effect of Hyder sine on Retinal Circulation

Sondermann, R. $\uparrow$, Die Entstehung des normalen Augen-

drucks 141

Stankovíc, M., Ein Fall von doppeltem Kayser-Fleischer-

schem Ring 100

Szauter, A., vide A. Fazakas.

Urrets-Zavalia (h.), A., Mode d'action de la cyclodiathermie 257

Verriest, G., vide/. Francois.

Vrabec, Fr., Sur une forme spéciale de revolution ultérieure

des précipités au cours de quelques uvéites chroniques . 26

- Description histologique d'un cas du psoriasis à localisation conjonctivale, cornéenne et cutanée .... 105

Vukovich, V., Das ERG des Achromaten

Wagenaar, J. W., Traumatic aniridia 193

- $\quad$ The Importance of the Relationship "Figure andGround" in Fast Traffic 309

Weekers, L., et R. Weekers, Traitement de Thypertension

oculaire par la diathermie rétrociliaire 221

et E. Prijot, Mesure de la resistance à Гécoulement de Thumeur aqueuse au moyen du tonomètre électronique. 5e partie. Mode d'action de Гiridencleisis dans le glau-come chronique non

inflammatoire 166

vide E. Prijot.

vide L. Weekers.

Weigelin, E., und H. Althaus, Unte $\Gamma$ suchungen an kreislauf-gesunden Personen über das

Verhalten des Netzhautarte-riendruckes beim Kältetest nach Hines und Brown . . 1

Weinstein, P., Nervism in Ophthalmology. Experiences of

Novocain Blockade of the Ciliary Ganglia .... 228

Wettler, H., Über einen Fall von Wernerschem Syndrom . 279

Zahn, K., vide M. Simková.

GESELLSCHAFTSBERICHTE - SOCIETY TRANSACTIONS - SOCIÉTÉS

57. Tagung der Deutschen Ophthalmologischen Gesellschaft vom 3. bis

5. September 1951 in Heidelberg 50, 112, 173

The Netherlands Ophthalmological Society. 122nd Meeting, held 19thand 20th May, 1951, in the Ophthalmological Clinic of the University Hospital, Leyden

PERSONALIA

Maximilian Salzmann - 90 Jahre alt $\quad 380$

NECROLOGIA

Hans Lauber $\dagger \quad 126$

In Memoriam Prof. J. van der Hoeve 190

BUCHBESPRECHUNGEN - BOOKS REVIEW - LIVRES NOUVEAUX

6.63

127

192

254 
316

379

VARIA

.64

255

320 